EPiC Series in Computing
Volume 78, 2021, Pages 126-135
Proceedings of the European Univer-
sity Information Systems Conference 2021

\title{
Coping with the COVID-19 challenges in a comprehensive university: learning tools and procedures adopted by Aristotle University of Thessaloniki
}

\author{
Vasiliki Kalfa $^{1}$, Georgios Roussos ${ }^{2}$, Dimos Charidimou ${ }^{3}$ and Angeliki \\ Agorogianni ${ }^{4}$ \\ ${ }^{1,2,3,4}$ IT Center - Aristotle University of Thessaloniki Greece \\ vasilikikeit.auth.gr, groudit.auth.gr, dharidimou@it.auth.gr, \\ aagorogieit.auth.gr
}

\begin{abstract}
In the context of the Covid-19 pandemic, learning rapidly has undergone a digital transformation into eLearning in order to cope with the circumstances of a global outbreak. This paper presents the use of synchronous and an asynchronous service both for teaching and assessment in one of the largest universities of Greece, Aristotle University of Thessaloniki (AUTh). The transition to eLearning has been achieved through a combination of commercial and open source software tools (mainly Zoom and Moodle). Moodle has reemerged in the AUTh academic community with improved functionalities from various plugins installed to meet the diverse range of pedagogical needs. It also describes the integration of Zoom Meetings solutions using centralized account authentication, i.e. single-sign-on (SSO) for the entire campus. End-user support and training is also provided as a combination of methods to support users on a large scale. We also present figures that highlight the high usage of eLearning systems with an added survey of positive users' opinion on how Aristotle University has gotten ahead with the pandemic and the architecture designed to balance the workload. Finally, we introduce current and future improvements.
\end{abstract}

\section{Introduction}

The first wave of Covid-19 pandemic reached Aristotle University (AUTh) in the beginning of Spring Semester 2020. Overnight, a vast campus of thousands of students and professors were faced with the radical transformation towards online learning. This emergency eLearning demanded swift adaptation of courses to an entirely new learning approach and awoke the need for the appropriate 
tools to provide an uninterrupted transition. The day-to-day activities of the academic community were among the first to begin this transition.

For distance learning, Moodle was already integrated as a supportive LMS platform for professors to use according to their educational needs on asynchronous teaching and learning. However, only a small percentage was skilled to extensively use the online tools available effectively for distance learning. The main use was registered as a repository of lecture presentations. Zoom, on the other hand, has been rated the most user-friendly tool for synchronous teaching.

Aristotle University was faced with the challenge of having to deal with a large multidisciplinary community interacting at an online level raising the issue of resources, tools and support. The team of IT administrators was skilled and experienced in the required systems and therefore, able to perform the necessary transition.

In this paper, we present the practices used on both Moodle and Zoom to support the AUTh academic community.

\section{The way to an asynchronous classroom}

\subsection{Lectures and online tools}

The main question remained as to which tool is the most effective to provide and support synchronous online lectures. Professors were assisted consequently in two ways, with using various video conferencing software packages (Zoom, Microsoft Teams, Skype for Business, Google Meet \& BigBlueButton) and with providing document cameras and pen displays to assist shared educational material. (Fig.1)

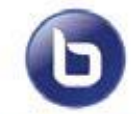

BigBluebutton

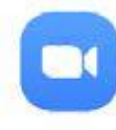

Zoom

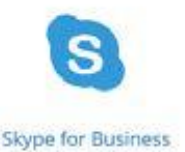

Skype for Business

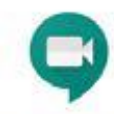

Google Meet

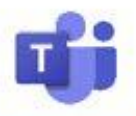

Microsoft Teams

Figure 1: Video conferencing software packages

Usage statistics show that the majority of the users chose the Zoom solution (Fig. 2). In any case, we should point out that the design of any type of distance eLearning should be based on pedagogical models-theories, teaching strategies and interactive learning technologies in order to ensure effective learning outcomes. University's Faculty Staff with a background in eLearning technologies provided quick guides for scenarios and best practices that could be followed by all Faculty Members.

\subsection{Student Assessment and Online exams}

The evaluation of learning outcomes should always take into account the different types of distance educational lesson-program (synchronous, asynchronous, video conferencing, hybrid, etc) and always in relation to the desired objectives and learning outcomes. AUTh represents a variation of educational programmes differing from human science studies to natural and applied science. This raises the issue of multiple and unique activities required by each department of studies. Moodle was used to cover the needs and support with the appropriate educational online tools that were integrated in the main system.

For engineering and natural science courses, algebraic and programming questions types were made available where students could submit a response either for assessing mathematical functions 
(Stack, Algebra) or programming codes (Coderunner). These tools were made available at the begin of the semester and therefore, professors were able to transform their assessment approach towards.

Online exams seem most challenging during the quick transition to e-learning. (Fig.2) It demands the proper tools to consider right validation on student assessment and minimize students cheating and reliability of students' own work. Identity validation was achieved through institutional SSO that is used in all of AUTh services to authenticate users. Further on, the quality of information that is demanded between student exam activity was therefore guaranteed via blocking concurrent sessions in quizzes or using the Safe Exam Browser as a safe web browser environment to control access to certain applications and prevent unauthorized use of the workstation used. Other e-proctoring tools were considered but dismissed due to students' data privacy reasons.

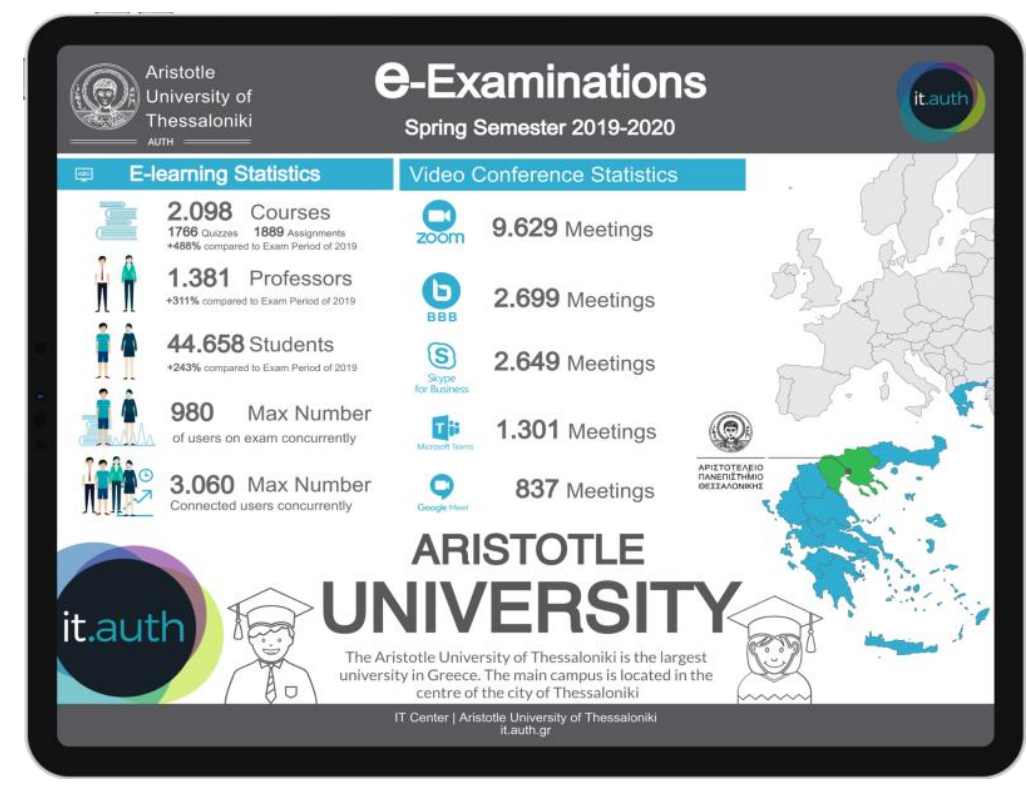

Figure 2: Statistics of spring semester 2020 exams and video conferencing sessions

\subsection{Student Assessment and Online exams}

Peer support was provisioned and achieved during the first months of the pandemic. Good practices and knowledge sharing were initiated to encourage professors with less experience on online distance learning. Moodle fora were used to ease communication on specific subjects varying from technical skills that a professor may need to opinions on tools and best-case learning approaches.

Guides and video tutorials were created from the administrators for training purposes of both students and professors (Fig.3). Demo meeting rooms were made available, especially for checking connection at any time prior to a lecture. 


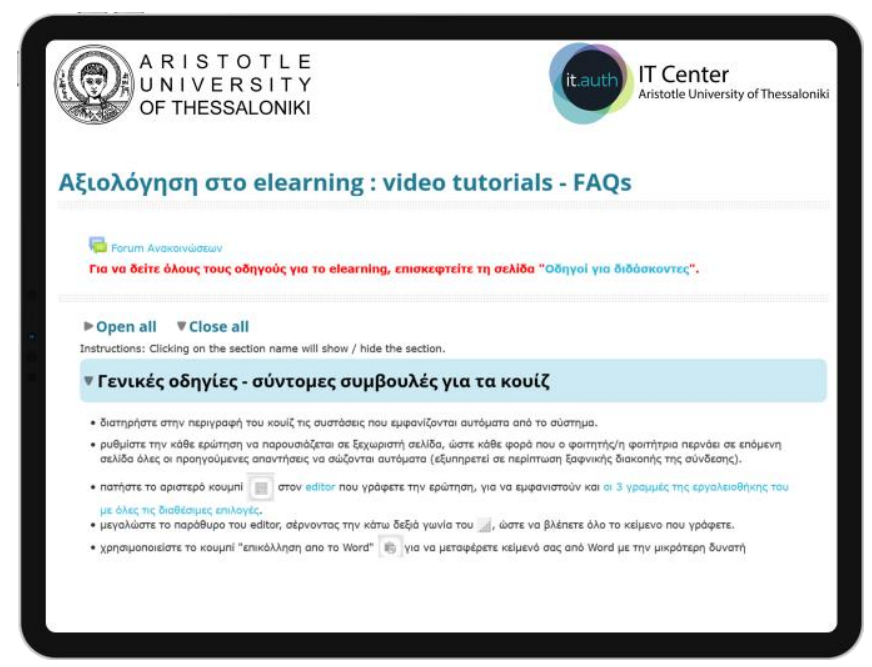

Figure 3: Video tutorials, FAQs in Greek language for student assessment on Moodle

\subsection{Student Assessment and Online exams}

To ensure uninterrupted access to the online platform a limit of maximum users allowed was stated during the semester and especially during the exams period. Considering this knowledge gained in the spring semester 2020, it was obvious that scaling out the Moodle installation was needed to be introduced to AUTh to alleviate the day-to-day activities of a vast academic community and carry the workload. An architecture of resources and analytics was introduced accordingly and equipped to balance the day-to-day load and the extreme exams load. (Fig.4)

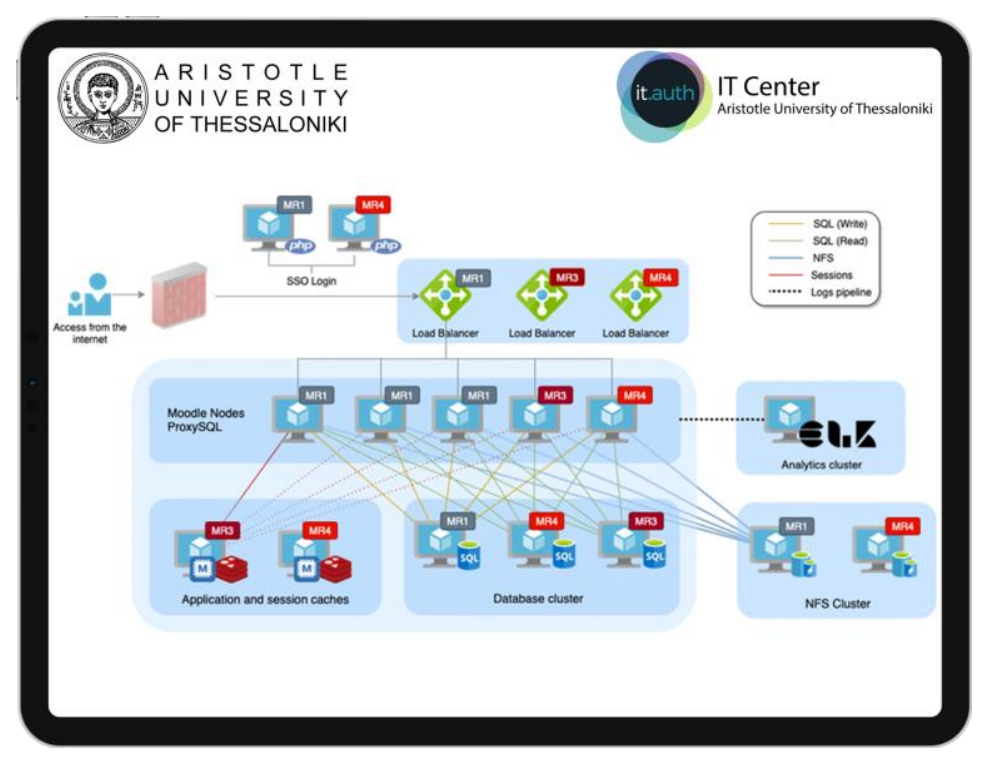

Figure 4: Architecture and deployment by the Computing Infrastructure Department of the IT Center 


\section{Synchronous classroom with Zoom, safeguarding academic community's credentials using SSO integration based on SAML 2.0}

The evolution of ICT technology and the promotion of direct communication and social interaction with both the real and the virtual world has created the idea that the world we live in is shrinking, compressing and coming closer both temporally and geographically. At the same time, events that take place simultaneously in different locations can reach the observer's frame of reference with immediacy and break the spatial and temporal barriers of the physical world, resulting in increased productivity as well as learning (Warf, 2011).

The searches that shaped 2020 according to Google Trends Platform were announced recently and "Coronavirus" was the No1 keyword in searches and "Zoom" was the No. 4 keyword globally (Google Trends website, 2020). Zoom was one of many tools for distance education that Aristotle University extensively used and implemented according to its needs, during the 2020 year. At Aristotle University we use the well-known Single Sign-On (SSO) which is a session and user authentication service. The use of SSO is widely considered as secured service that permits a user to use one set of login credentials eg. username and password to access multiple applications inside and outside of the university.

In addition, SAML is a standard protocol used by world's most famous web browsers to enable Single Sign-On (SSO) through secure tokens. The great thing regarding SAML is that it completely eliminates the need for passwords since this technology uses standard cryptography and digital signatures to pass a secure sign-in token from an identity provider (Aristotle University) to a SaaS application (Zoom).

\subsection{How Zoom and SSO integration works in our University}

As we know, Zoom is a cloud-based video communications app that allows you to set up virtual video and audio conferencing, webinars, live chats, screen-sharing, and other collaborative capabilities named as a leader in the 2020 Gartner Magic Quadrant Report (Zoom website, 2020). Specifically, it is a Software as a Service (SaaS) that provides a variety of integration capabilities with other platforms, applications and tools like SSO authentication as mentioned.

As regards the Zoom side, Zoom acts as the Service Provider (SP), and offers automatic user provisioning. Having this in mind, we don't need to register as a user in Zoom. Once the Zoom system receives a SAML response from the Identity Provider (IdP), Zoom system checks if this user exists or not. If the user does not exist, Zoom system creates a user account automatically with the received name ID.

In a basic scenario the web SSO service, actually is an agent module on the application server retrieves the specific authentication academic credentials for an individual academic user from our dedicated SSO policy server, while authenticating the user against a user repository, such as a Lightweight Directory Access Protocol (LDAP) directory. (Fig.5)

As a result, the SSO service authenticates the academic user to log in once and access services without re-entering authentication factors. In other words, an academic user is authenticated for all the applications the student / Professor has been given rights to and eliminates future password prompts for individual SaaS applications during the same session. 


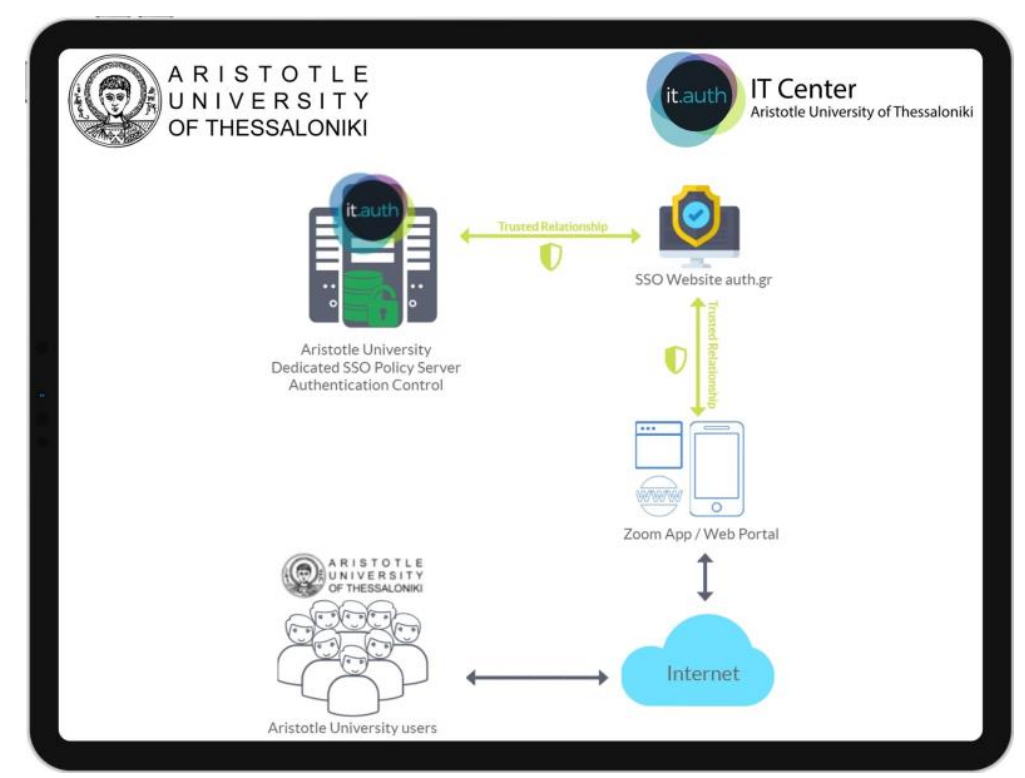

Figure 5: SSO schema for Zoom use

All sensitive credentials like academic email, username and password are encrypted (hash text) and in any case not stored in Zoom's servers but in Aristotle's University data center. Whether in paper, electronic or any other format, records of personal information must be safeguarded at all times. SSO integration based on SAML 2.0 was one of the most reasonable steps to protect academic data against theft, loss or unauthorized collection, use, disclosure, copying, modification, disposal etc.

Manual configuration of SSO through Zoom platform was not a complex process. In fact it was as easy as $\mathrm{ABC}$, since Aristotle University uses SSO authentication in a variety of web applications and platforms. What was the settings procedure?

\subsection{Manual configuration of the SSO through Zoom's web portal}

Firstly, we configured our IDP to send all the necessary elements to Zoom, actually any unique identifier. Secondly, though zoom.us web portal we configured SSO SAML basic settings like Sign-in Page URL, Sign-out Page URL, Identity Provider Certificate, Service Provider (SP) Entity ID, Issuer (IDP Entity ID) and after some other security settings. The following figure shows the settings in detail. (Fig.6)

Afterwards, we set up the values and attributes of the SAML response mapping and we did some advanced information mapping concerning to which users of our community will obtain a Zoom license or not. In our case we configured to give automatically zoom licenses only to Teaching and Administration Stuff by reading the specific SAML Attribute and value (Job Title). (Fig.7)

Since Aristotle University is under GDPR law, to comply with the data privacy regulations, in terms of safeguarding the data, being transparent, we have to know exactly what data we hold and what data we send outside in this case to Zoom. Since safeguarding our academic community's credentials has been and always will be one of the most important security goals for the whole IT team, we constantly try to eliminate the possibility of any future threats and risks associated with the manner in which the information is kept in any system or app we use. 


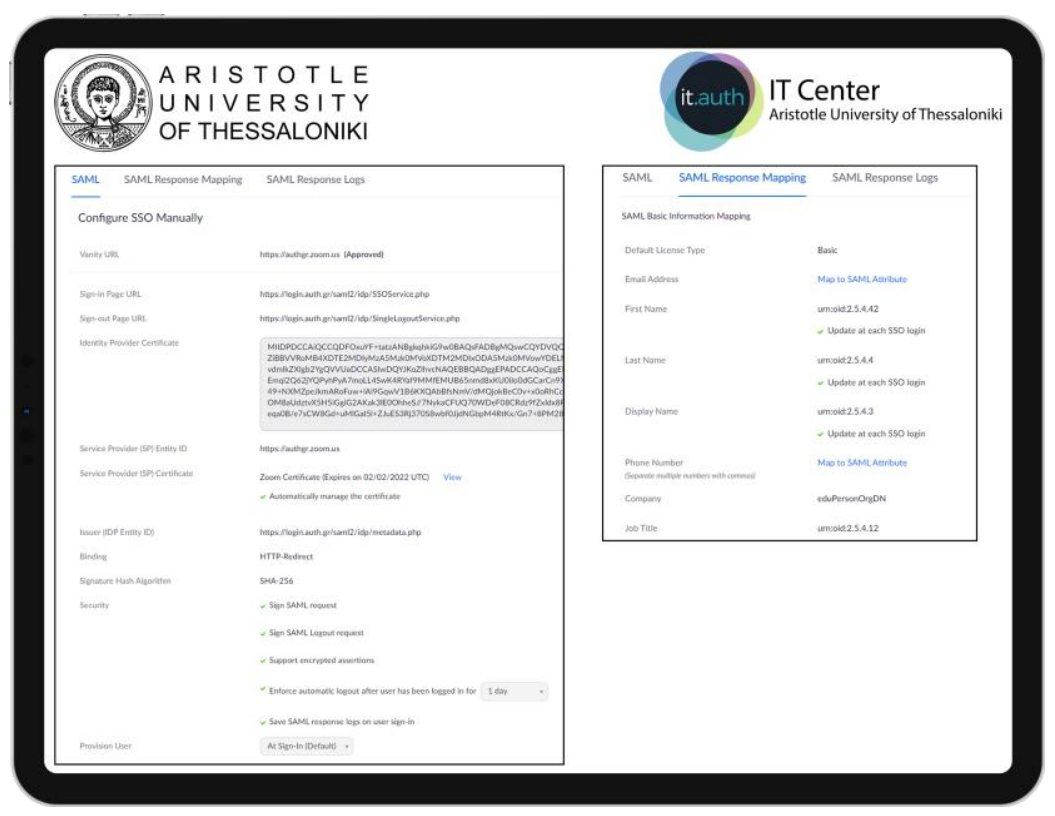

Figure 6: Manual Configuration of SSO through Zoom web portal

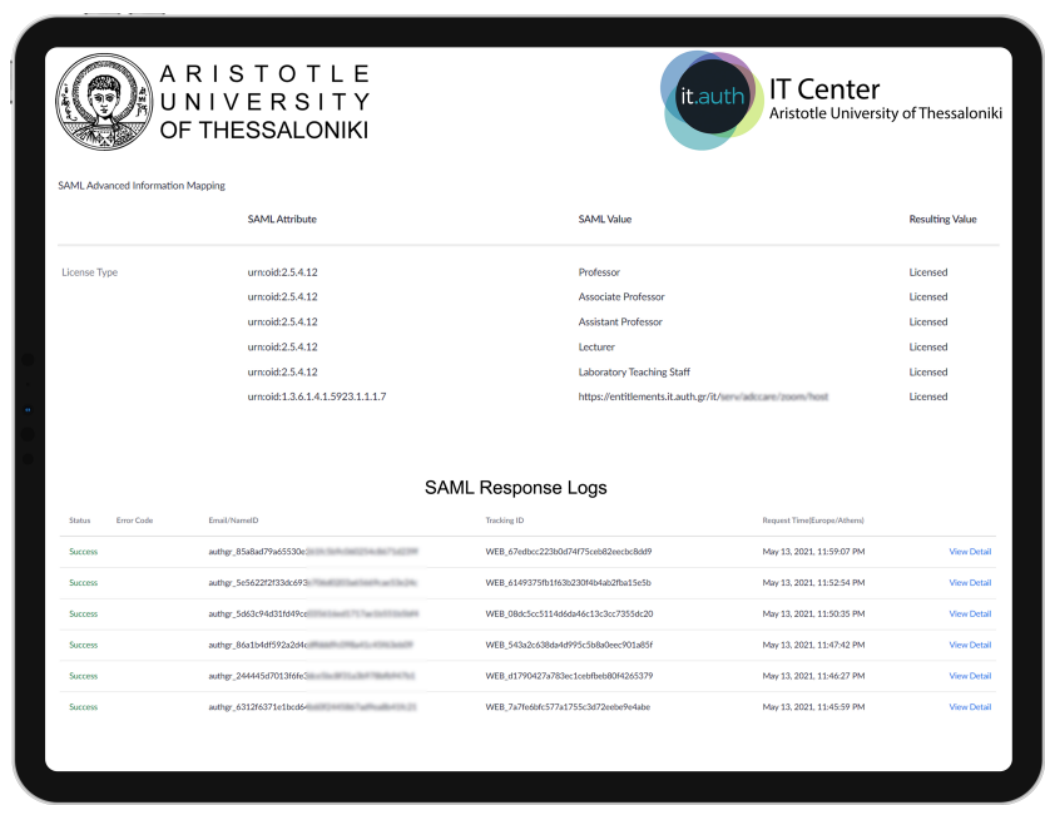

Figure 7: SAML Advanced Information Mapping and Response Logs 
For that reason, IT Center of AUTh selected SSO which is much simpler, more convenient and secure for academic users. However, this may seem counterintuitive when we think how single-signon is better, instead of multiple sign-in times with multiple different passwords, be more secure in any use scenario. Here are some benefits in practice:

- Better password policy enforcement

- Mitigate risk for access to 3rd-party sites. Academic credentials (email, user, passwords) are not stored or managed externally.

- Reduce password fatigue from different username and password combinations

- Reduce time spent re-entering passwords for the same identity

- Reduce IT costs and less time wasted on password recovery

- Word documents (.docx), which can be produced by Microsoft Word.

\subsection{Annual Usage Statistics}

In practice, it seems that the majority of users in our academic community adopted this video conference tool and this specific authentication method easily and quickly once again. As the following figure shows (Fig.8), more than 46K users are using Zoom platform daily and now more than $241 \mathrm{~K}$ meetings are established per month (February 2021: 170K meetings, March 2021: 210K meetings, April 2021: 241K meetings). Moreover, according to Zoom's usage charts, users are flexible to use Zoom with SSO using any type of device without any major problem.

On the other hand, after one year of use, we never faced any issue regarding the safety of sensitive data of our University users. This clearly proves that not only we do have the complete control over the whole academic user data, but we also have much more time and funds to improve or develop apps for educational purposes especially regarding eLearning.

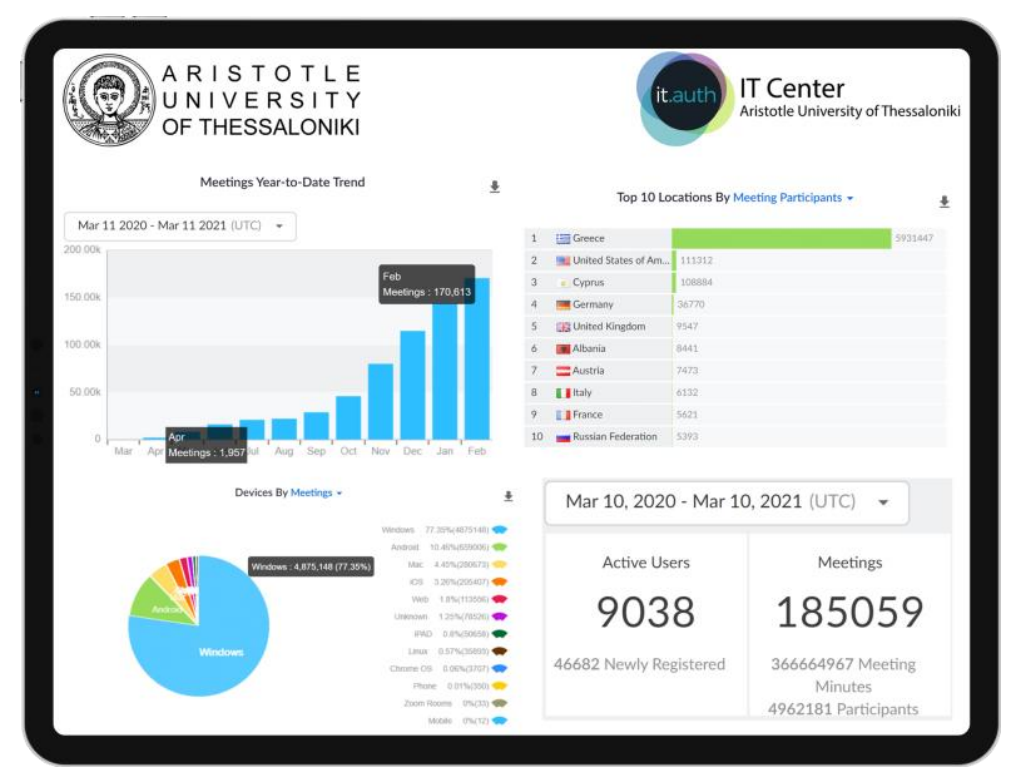

Figure 8: Annual statistics of Zoom use from the beginning of the Pandemic onwards 


\subsection{User Satisfaction Survey for IT Services during pandemic.}

Every year IT Center of Auth organizes and conducts user satisfaction surveys in order to continuously improve its services. In the annual survey of 2020 involved 8,226 participants, which is the highest number of participants of all time.

As it seems there is a positive satisfaction among our users based on their opinion on how the Aristotle University has gotten ahead with the pandemic until now (Fig.9). The findings of this recent survey provided to us valuable insights to set our next goals and future steps still in the midst of a pandemic.

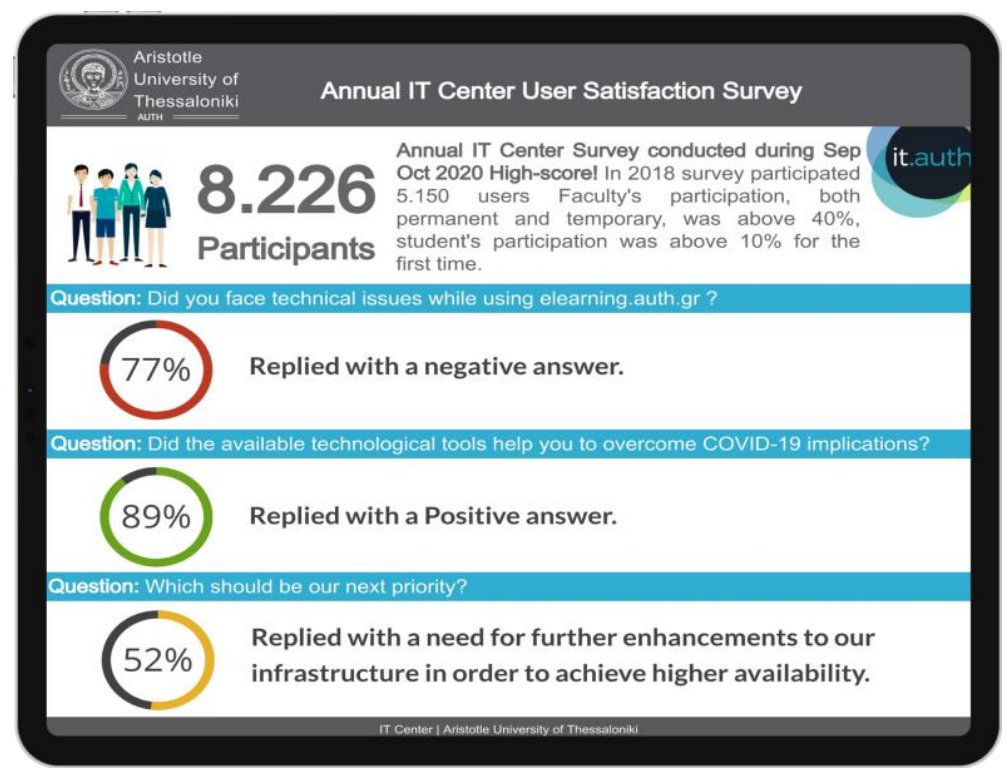

Figure 9: Survey results on user satisfaction

Following this optimistic message from our academic community, it is necessary to continue the increase of quality of the services provided taking into account the EDUCAUSE report about the future of hybrid learning after the pandemic. This specifically translates to further work with respect to pedagogical and technological aspects for the full exploitation of the benefits of this large-scale rapid adoption of eLearning (Grajek S. \& Educause Report, 2020).

\section{New features \& Future steps}

A new feature was implemented and will be widely available to users in relation to Moodle Zoom connection through the LTI Pro installation. This will enable a direct collaboration of both systems and thus, minimizing user interaction with duplicate steps of workflow.

Future steps contain refinements on the existing services provided in order to become more userfriendly and universally support accessibility. For example, a point worth mentioning is a new CMS is currently underway to create a user-friendly interface for guides and tutorials. 


\section{Adding Figures and Tables}

COVID-19 has changed the world, and academia is no exception. Since many academic institutions all over the world promptly shifted all educational activities to the e-learning format, now are realizing that obviously teaching practices will ultimately never completely return to the previous model. According to all the facts and figures presented in this paper Aristotle University reacted in an effective manner due to the existed centralized IT services and the addition of tools in a timely manner from the beginning of the pandemic. Even in the midst of the COVID-19 pandemic and even if that has changed education forever, we are focused on providing technology efficient way to support the use of synchronous and asynchronous services both for teaching and assessment while protecting the sensitive data of all users in our academic community. Overcoming challenges and hardships posed by the onogoing pandemic, IT Center of AUTh is coping with the new normal and quickly moves forward by planning educational tech investments.

\section{References}

Carlisle, D. (2010, April). graphicx: Enhanced support for graphics. Retrieved from http://www.ctan.org/tex-archive/ help/Catalogue/entries/graphicx.html

Voronkov, A. (2004). EasyChair conference system. Retrieved from easychair.org

Voronkov, A. (2014). Keynote talk: EasyChair. In Proceeedings of the 29th ACM/IEEE International Conference on Automated Software Engineering (pp. 3-4). ACM. Retrieved from http://dl.acm.org/citation.cfm?id=2643085\&dl=ACM\&coll=DL

Voronkov, A., \& Hoder, K. (n.d.). Templates. Retrieved from Templates for proceedings: https://easychair.org/proceedings/template.cgi? $\mathrm{a}=12732737$

Wikipedia. (n.d.). EasyChair. Retrieved from Wikipedia: https://en.wikipedia.org/wiki/EasyChair 